\title{
Effects of visual fatigue on EEG features
}

\author{
Xu Junli, Mu Zhendong \\ College of Information Engineering, Jiangxi College of Technology, Jiangxi Nanchang 330098
}

Keywords: Visual evoked potentials; Fatigue; EEG

\begin{abstract}
VEP is a major tool for EEG studies, this paper is the use of visual evoked potentials identification study, a study of the visual impact of fatigue on the identification results, this paper ten subjects EEG acquisition, respectively, and let them do the same patterns of brain electrical repeatedly obtain experiment, every two days to do an experiment to study the classification accuracy rate of change, the end result is displayed as after many experiments, the classification accuracy will decrease.
\end{abstract}

\section{Introduction}

Event-related potentials (ERP) is a special kind of cerebral evoked potentials, by intentionally imparting a special psychological significance stimulate, or the use of a plurality of potential brain caused by a variety of stimuli. It reflects the cognitive process of neurophysiologic changes in the brain. Visual evoked potentials in the EEG study, are widely used, early Eric Courchesne, Steven A Hillyard, Robert Galambos et al., 1975 digital, geometry and other visual stimuli as a point, through research and other related visual P300 brain waves components of the physiological and psychological analysis of relevant related brain, which began using event-related potentials EEG analysis.

A VEP EEG feature with other EEG stability is missing an advantage, and a stability characteristic of the BCI system is to ensure the accuracy of the main factors. The so-called brain-computer interface is the use of brain waves to control external devices a new type of technology. An event evoked potential BCI research and analysis steps can be summarized as follows: identify research purposes -> design experiments EEG acquisition mode feature extraction $->$ Result analysis concluded result of applying. Today's research focused on feature extraction and analysis of the results, such as the use of visual evoked potentials Kapeller $\mathrm{C}$ were the brains of the audio interface controlled studies, a subject through visual evoked potential studies, the use of steady-state visual evoked potential as tools, the use of existing classifier feature analysis, classification accuracy rate of $99.21 \%$, as described in the literature, are using P300 brain-computer interface research, experimental design mode, and then collect the EEG feature extraction and analysis made the corresponding research.

And feature extraction studies, compared to the visual evoked potential stimulation model for the results of the impact studies are rare, experimental results are also rare. Regan D, et al for the VEP visual fatigue were studied, Regan team studied during the experiment, the effect of the experimental subjects long visual fatigue caused by the characteristics of the EEG, although it inspired visual fatigue impact characteristics, but for long-term concern subject to the same object, whether EEG feature enhancements or decay did not give relevant results. Based on visual evoked potentials during the identification process of research, we have designed a variety of experimental models and 
found that the different experimental models have an impact on the results, this paper is mainly aimed at individuals are under the same experimental model, every two days to do one experiment, for a month, the impact on the identification of EEG results.

\section{Method}

Step 1, Block larger drift EEG: in EEG acquisition process, such as the subjects movement, wander, outside sound effects, the EEG signal initial there will be large drift, will follow the EEG signal processing impact, so in the EEG before treatment, to remove this a part of the brain electrical signal;

Step 2, Ocular artifact reduction: the EEG signal in the original, because to blink or look right and left, the impact on the eye electric signal, so before feature extraction and classification, to remove the impact of this part, this paper is mainly to remove the vertical eye film;

Step 3, epoch the data, view the stimulus intervals, and generally $10 \%-20 \%,-50$, is the common value of -100; the spirit of not more than one event to the principle, in this paper, the interception of data in -100 900ms.

Step 4, baseline correction: the segmented data many not at baseline, so this paper conducted a total of two times the baseline correction and a linear correction.

Step 5, artifact reject: EEG signals collected by the class, there is a part of segmented data caused by various reasons is not good, not only for data analysis useless, it will affect the analysis of the data, so to choose a certain window screening, the window is $-80 \sim 80$.

Step 6, average: This article mainly is ERP analysis of EEG signals, so the same stimulation of the brain electrical signal types were stacked.

After obtaining a segmented EEG available, we use the Fisher distance to extract the features of visual fatigue.

The Fisher distance was often used to denote differences between classes in classification research. The bigger the fisher distance was the more notable the difference was.

The Fisher class reparability criterion was used preparatory to extract features. The Fisher distance of two classes was calculated as

$$
F=\frac{\left(\mu_{1}-\mu_{2}\right)^{2}}{\sigma_{1}^{2}+\sigma_{2}^{2}}
$$

Where $\mu$ was equalizing value and $\sigma$ was variance.

\section{Result}

Whether visual fatigue will affect the EEG features? We are on the same subjects in the same experimental model of EEG acquisition, and then using the same methods for data analysis, comparative analysis of experimental results characteristic change. In Figure 1 shows the trend of EEG signal in the time domain. 


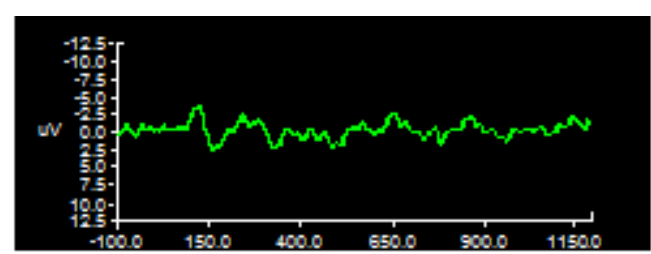

a

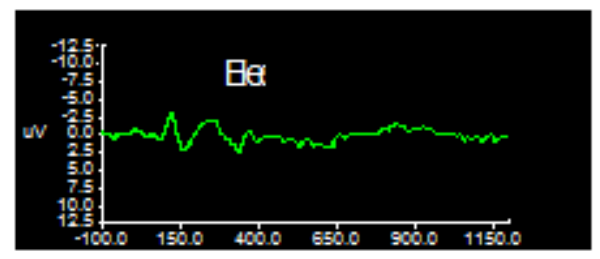

$\mathrm{c}$

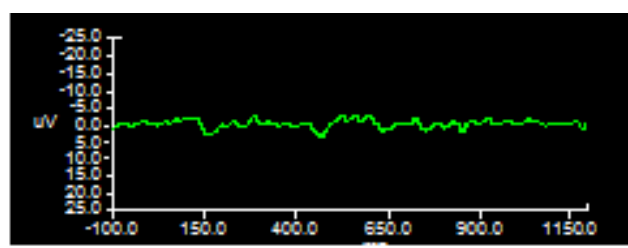

b

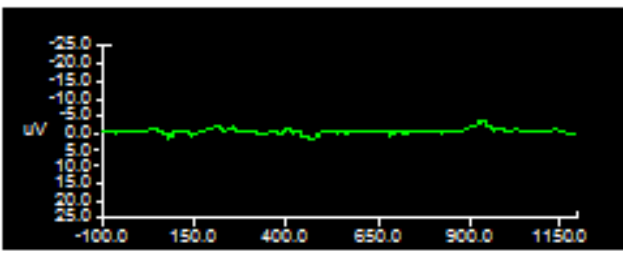

d

Figure. 1 Contrast affects the time domain

Figure 2 (a) is the first experimental results, FIG. 2 (b) made after the results of the two time (interval of two days each experiment, after 4 days' intervals twice), Figure 2 (c) show done results after 4 times, FIG. 2 (c) show the results of experiments done six times, FIG. 2 (d) show the results of eight times done experiments.

The results show that there are some long-term experiments on brain signals, and Figure 2 shows the effect of different times EEG classification accuracy rate.

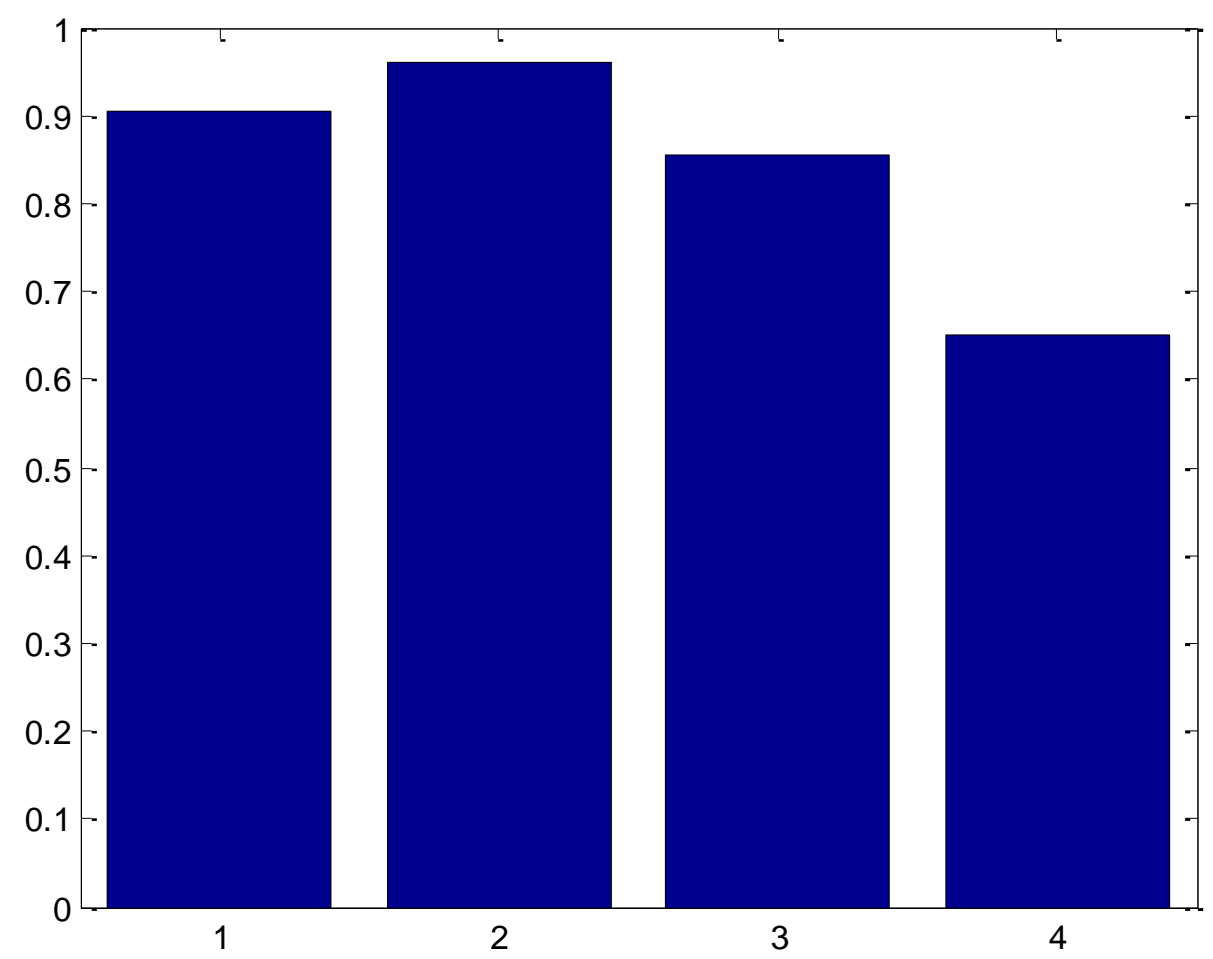

Figure. 2 results of compare the accuracy

Figure 2 is the $\mathrm{x}$-axis of the test four times, y-axis shows the accuracy. From comparison of accuracy, accurate first to have a rise, then fall sharply, indicating that many experiments began to have a strengthening effect on the features, but with the increase in the number of experiments, the fatigue began to show characteristics begin to weaken. 


\section{Conclusion}

VEP is an important tool in EEG research, but research on the visual experiment itself is rarely the subject of this article from several experiments EEG impact analysis to verify the classification results from the identification, the results after many experiments show, the subject will produce visual fatigue, which can affect the fatigue characteristics of the subject.

\section{Acknowledgements}

This work was financially supported by project of Technology Department of Jiangxi Province [No 20143BBM26048] and project of Jiangxi University of Technology [No. xtcx201312].

\section{References}

[1]Lambooij M, Fortuin M, Heynderickx I, et al. Visual discomfort and visual fatigue of stereoscopic displays: a review[J]. Journal of Imaging Science and Technology, 2009, 53(3): 30201-1-30201-14.

[2]Li H C O, Seo J, Kham K, et al. Measurement of 3D visual fatigue using event-related potential (ERP): 3D oddball paradigm[C]//3DTV Conference: The True Vision-Capture, Transmission and Display of 3D Video, 2008. IEEE, 2008: 213-216.

[3]Murata K, Araki S, Kawakami N, et al. Central nervous system effects and visual fatigue in VDT workers[J]. International archives of occupational and environmental health, 1991, 63(2): 109-113.

[4]Yijun W, Ruiping W, Xiaorong G, et al. Brain-computer interface based on the high-frequency steady-state visual evoked potential[C]//Neural Interface and Control, 2005. Proceedings. 2005 First International Conference on. IEEE, 2005: 37-39.

[5]Murata A, Uetake A, Takasawa Y. Evaluation of mental fatigue using feature parameter extracted from event-related potential[J]. International Journal of Industrial Ergonomics, 2005, 35(8): 761-770. 